\title{
Design of Dual-Bandpass Hybrid Waveguide-Microstrip Microwave Filters
}

\author{
Mónica Martínez-Mendoza, Student Member, IEEE, Juan Sebastián Gómez-Díaz, Student Member, IEEE, \\ David Cañete-Rebenaque, Student Member, IEEE, and Alejandro Alvarez-Melcón, Senior Member, IEEE
}

\begin{abstract}
A simple dual-bandpass filtering structure is proposed. The filter is implemented by means of a new hybrid waveguide-microstrip technology. The novelty of the structure is that it combines two standard printed microstrip coupled line resonators, with a resonance of the base waveguide cavity, to implement a dual-band operation. Important aspects about the filter design, useful for practical applications are treated. This includes a study on the maximum coupling that can be achieved to the cavity resonance, and guidelines to control the positions of the two passbands and the transmission zeros. Besides, an example has been designed, manufactured and tested. The measurements on the fabricated prototype have confirmed the validity of the new structure to perform dual-band operation.
\end{abstract}

Index Terms-Dual-band filter synthesis, dual-band filters, hybrid waveguide-microstrip technology, microstrip resonators, microwave filter.

\section{INTRODUCTION}

$\mathbf{T}$ HE PROBLEM of the synthesis and design of microwave dual-bandpass filters has been treated in several recent contributions using different approaches. By way of illustration, two synthesis and design techniques for filters with one stopband located between two passbands were introduced in [1]. The first technique allows to synthesize two passbands with symmetric frequency characteristics. The second method allows to obtain two passbands with different widths, but with the stopband determined by $(n / 2)$ transmission zeros all placed at the same frequency, being $(n)$ the order of the filter. A different approach can be found in [2], where a transformation from a regular single-passband low-pass prototype to another with two sub-bands was for the first time presented. Furthermore, a different type of approaches for the synthesis of multibandpass filters are those based on optimization. A very interesting contribution, which employs the coupling matrix of single-band responses, with subsequent optimization of its entries to design dual and triple-band filters, was proposed in [3]. Besides, a technique that provides all the possible solutions of a given topology was presented in [4]. This technique is applicable to multiband

Manuscript received February 20, 2008; revised August 15, 2008. First published November 18, 2008; current version published December 05, 2008. This work was supported by the Centro de Investigación Cientifica y Tecnológica (CICYT) under Spanish National Project TEC2007-67630-C03-02, and under Regional Seneca Project 02972/PI/05.

The authors are with the Telecommunication and Electromagnetic Group, Technical University of Cartagena, Cartagena E-30202, Spain (e-mail: shanaz00@hotmail.com; alejandro.alvarez@upct.es).

Color versions of one or more of the figures in this paper are available online at http://ieeexplore.ieee.org.

Digital Object Identifier 10.1109/TMTT.2008.2007189 filters within the narrow-band approximation. Finally, a technique presented in [5] guarantees the convergence during the computation of the coupling matrix for any multiband filtering characteristic, of a novel iterative algorithm.

In this paper, a new implementation of a simple dual-bandpass filtering configuration is introduced, based on a hybrid waveguide-microstrip technology. The hybrid technology consists of a compact structure combining the waveguide and the microstrip technologies, first introduced in [6]. However, only bandpass transversal filters have been implemented with this innovative structure until now. In this contribution we propose the employment of a different coupling topology to implement, for the first time, a dual-bandpass hybrid waveguide-microstrip filter.

The synthesis process is composed of several steps. First, we determine the prototype function with the idea of piecing together the functions of different individual filters, as presented in [3]. Next, we apply the direct synthesis technique introduced in [7] to obtain an initial coupling matrix, which will then be used in an optimization process (see [4], [5], and [8]) to transform the initial coupling matrix into a matrix, which represents the desired coupling topology of the filter. An important feature of the final obtained coupling matrix is that only one negative coupling is required. Therefore, it is suitable to be implemented using the hybrid waveguide-microstrip technology. In this technology, the change in sign of the electric field of the cavity resonance implements one negative coupling [6]. All other couplings to the printed microstrip resonators are considered positive. With all these considerations, the dimensions of the hybrid structure are optimized to implement the obtained coupling matrix. Good agreement is obtained between the response of the coupling matrix, results obtained through full wave simulations, and measurements, validating the new structure for dual-bandpass operation.

\section{TOPOLOGY DESCRIPTION}

This contribution is focused on the implementation of a dualbandpass filter by means of an innovative configuration based on the hybrid structure introduced for the first time in [6]. The new configuration is shown in Fig. 1. The structure is composed of two open line printed microstrip resonators, which are mutually coupled by proximity. They are printed between the input and the output ports, also implemented with printed microstrip lines. These microstrip resonators are combined with a waveguide resonance in order to implement the third-order coupling topology shown in Fig. 2. It can be noticed that the inter-resonator coupling between the two printed line microstrip resonators is implemented as a standard side parallel coupled lines section. 


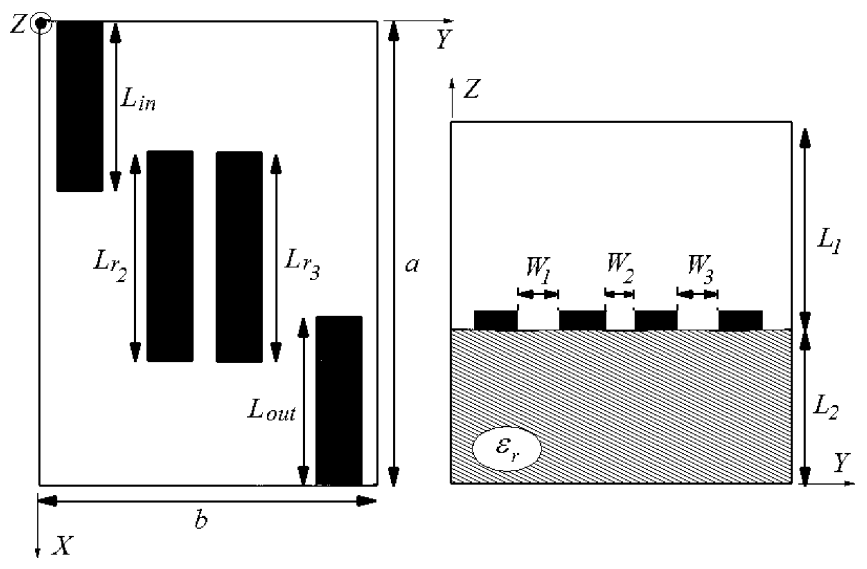

Fig. 1. Hybrid waveguide-microstrip structure for a third-order dual-bandpass filter following the coupling topology shown in Fig. 2. The width of the microstrip resonators, and of the input/output ports in the examples of this paper is $2 \mathrm{~mm}$.

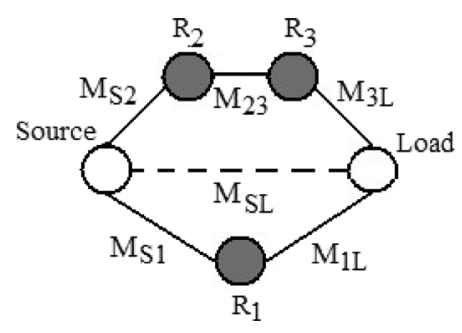

Fig. 2. Coupling scheme of a third-order fully canonical filter. $M_{S L}$ represents the direct coupling from source to load.

TABLE I

COUPLING MATRIX OF THE TOPOLOGY FOR THE DUAL-BANDPASS DESIGN

\begin{tabular}{|c|ccccc|}
\cline { 2 - 6 } \multicolumn{1}{c|}{} & $\mathrm{S}$ & 1 & 2 & 3 & $\mathrm{~L}$ \\
\hline $\mathrm{S}$ & 0 & $M_{S 1}$ & $M_{S 2}$ & 0 & $M_{S L}$ \\
1 & $M_{S 1}$ & $M_{11}$ & 0 & 0 & $M_{1 L}$ \\
2 & $M_{S 2}$ & 0 & $M_{22}$ & $M_{23}$ & 0 \\
3 & 0 & 0 & $M_{23}$ & $M_{33}$ & $M_{3 L}$ \\
$\mathrm{~L}$ & $M_{S L}$ & $M_{1 L}$ & 0 & $M_{3 L}$ & 0 \\
\hline
\end{tabular}

The $(N+2)$ by $(N+2)$ coupling matrix of this third-order configuration presents the form shown in Table I. In the hybrid structure, the mode excited in the partially filled cavity behaves as resonator $1\left(R_{1}\right)$. Furthermore, the structure includes resonators 2 and $3\left(R_{2}, R_{3}\right)$, which are coupled together. These resonators will be implemented in the hybrid structure with the two microstrip printed lines shown on the left side of Fig. 1. These two printed line resonators are sided coupled in order to implement the $M_{23}$ term of the coupling matrix. Finally, the hybrid structure implements a direct coupling between source and load, represented in Fig. 2 by $M_{S L}$.

As recognized in [6], the change in sign of the electric field associated to the waveguide mode from the input to the output port is responsible for the existence of a negative coupling. Therefore, the coupling term corresponding to resonator $R_{1}$ will satisfy the relation $M_{S 1}=-M_{1 L}$. No other negative coupling term is present in the matrix. This coupling configuration makes the dual-band design with the hybrid-structure of Fig. 1 possible, as it will be shown in the next sections. Note also that,

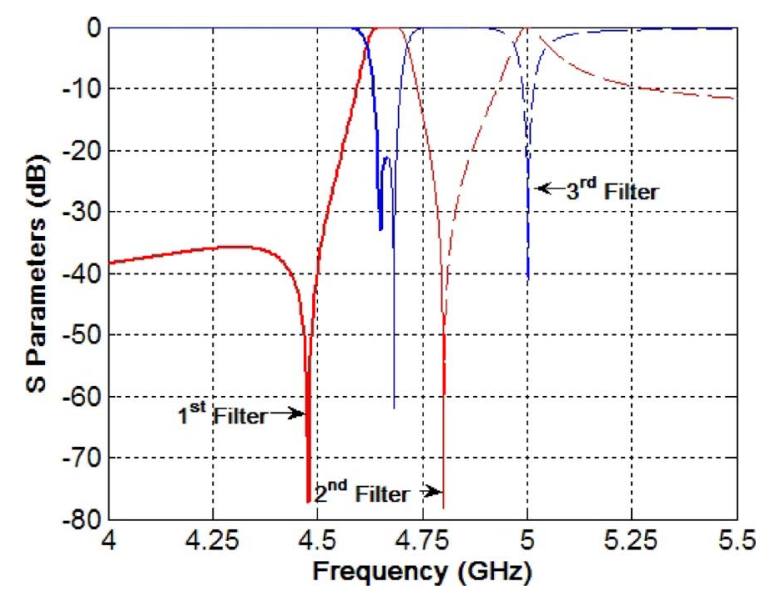

Fig. 3. Dual-bandpass prototype function, obtained by piecing together individual filters.

when symmetry is employed, the coupling terms will satisfy $M_{S 2}=M_{3 L}$ and $M_{22}=M_{33}$. The requirement of symmetry is not needed in the topology, but will simplify the design and optimization tasks of practical transfer functions.

\section{Design Example AND PRactical Issues}

In this section, important aspects about the filter design, useful for practical implementations, are studied. Besides, we are going to present an implementation example employing the innovative hybrid structure introduced for the first time in [6], but with the new configuration shown in Fig. 1 for the implementation of dual-band responses.

The synthesis method requires the computation of a prototype function to start with. To carry out this step, we use the approach introduced in [3], where the functions of individual filters were separately designed according to [9]. Then, they are put together to compose the final prototype function. Our design example consists of a dual bandpass filter with $-21 \mathrm{~dB}$ of return loss, and three transmission zeros at $1.5,4.5$, and $4.8 \mathrm{GHz}$. Its center frequency is $4.8 \mathrm{GHz}$ and its whole bandwidth is $362 \mathrm{MHz}$. The lower passband has two poles, whereas the upper passband has one pole (network of order 3 ). The first step of the design procedure will let us to determine the exact position of these three poles. The obtained prototype function is shown in Fig. 3, where the three individual filters used to compose the whole filtering function can be clearly distinguished. After the synthesis of the individual filters, the poles of the prototype function, required for the synthesis of the final filter, can be easily calculated. In our example the poles are found to be placed at 4.650, 4.682, and $5.002 \mathrm{GHz}$. Once the poles and the transmission zeros have been found, the numerator polynomials $F(s)$ and $P(s)$ of the scattering parameters can be calculated as follows [10]:

$$
S_{21}=\frac{P(s)}{\varepsilon \cdot E(s)} \quad S_{11}=\frac{F(s)}{\varepsilon_{R} \cdot E(s)} .
$$

The value of the constant $\varepsilon$ is adjusted to obtain $-21 \mathrm{~dB}$ return loss. Besides, with $F(s)$ and $P(s)$, the denominator polynomial $E(s)$ can be found using the alternating pole singularity principle, as mentioned in [10]. Thereby, we have totally determined the transfer and reflection scattering parameters $S_{11}(s)$ 
and $S_{21}(s)$, and the transversal coupling matrix can be synthesized with the procedure described in [7], obtaining

$$
M_{1}=\left(\begin{array}{rrrrr}
0 & 0.3042 & -0.3140 & -0.3162 & 0.0027 \\
0.3042 & 1.1136 & 0 & 0 & 0.3042 \\
-0.3140 & 0 & -0.9918 & 0 & 0.3140 \\
-0.3162 & 0 & 0 & 0.6687 & 0.3162 \\
0.0027 & 0.3042 & 0.3140 & 0.3162 & 0
\end{array}\right) .
$$

However, the implementation of this coupling matrix with the hybrid waveguide-microstrip technology is difficult, mainly because two negative couplings are required between the input/ output ports and two of the resonators. A more convenient coupling topology to implement the dualband response employing the hybrid technology is the previously described configuration of Fig. 2.

In order to compute a coupling matrix that represents the topology proposed in Fig. 2, we can use optimization techniques (see [4], [5], [8]). These techniques produce several possible solutions for the topology under study. One possible solution is given by the following coupling matrix $\left(M_{2}\right)$ :

$$
M_{2}=\left(\begin{array}{rrrrc}
0 & -0.3091 & 0.4227 & 0 & 0.0024 \\
-0.3091 & 0.6699 & 0 & 0 & 0.3091 \\
0.4227 & 0 & 0.0482 & 1.0432 & 0 \\
0 & 0 & 1.0432 & 0.0482 & 0.4227 \\
0.0024 & 0.3091 & 0 & 0.4227 & 0
\end{array}\right) \text {. }
$$

An alternative but equivalent coupling matrix can also be obtained. This alternative matrix results to be

$$
M_{3}=\left(\begin{array}{rrrrr}
0 & -0.3091 & 0.4227 & 0 & 0.0024 \\
-0.3091 & -0.9914 & 0 & 0 & 0.3091 \\
0.4227 & 0 & 0.8828 & 0.2078 & 0 \\
0 & 0 & 0.2078 & 0.8828 & 0.4227 \\
0.0024 & 0.3091 & 0 & 0.4227 & 0
\end{array}\right) \text {. }
$$

It is important to note that the inter-resonator coupling term $M_{23}$ is much smaller in the coupling matrix $M_{3}$ $\left(M_{23}=0.2078\right)$ than with the first option given by the matrix $M_{2}\left(M_{23}=1.0432\right)$. This can make the $M_{3}$ coupling matrix more interesting for practical purposes, specially when high coupling values are difficult to implement. Besides, the solution $M_{3}$ indicates that the resonance associated to the sign change implements the upper passband of the filter (for the resonator $R_{1}$, the diagonal element $M_{11}$ is the lowest value of all the diagonal elements). In the hybrid structure, the cavity mode will implement this resonance, and therefore it will take care of the upper passband of the filter. The remaining two coupled resonators $\left(R_{2}\right.$ and $R_{3}$ in Fig. 2) will be implemented by the parallel coupled microstrip lines, and will form the second-order lower passband.

It can be observed that these properties are not shared by the matrix $M_{2}$. In fact, the matrix $M_{2}$ combines the resonance that changes sign in the lower passband with one of the other coupled resonances. In the hybrid structure this requires the combination of the cavity mode with one of the microstrip line resonators, making more complex the design and optimization procedure of the structure. For these reasons, the next designs will be based on the solution given by the coupling matrix $M_{3}$.

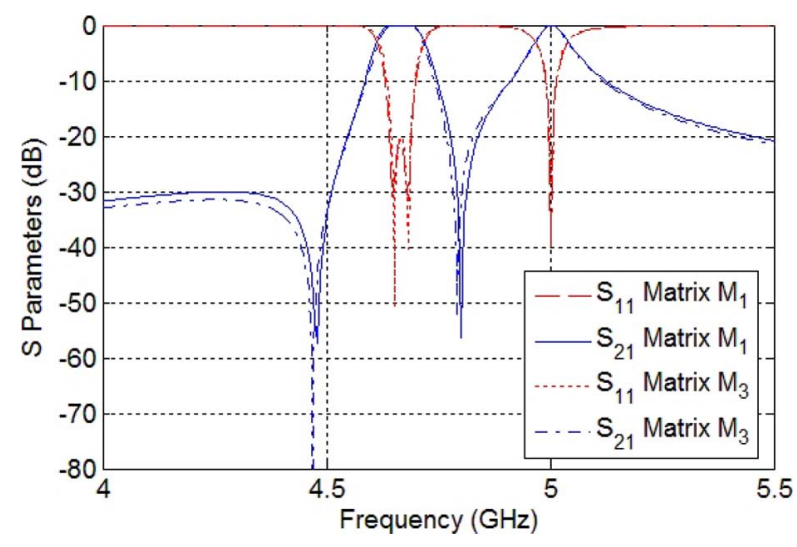

Fig. 4. Response of the coupling matrix $M_{3}$ versus the original prototype function (matrix $M_{1}$ ).

In addition, it is important to remark that it is possible to implement the final coupling matrix $M_{3}$ with the hybrid structure shown in Fig. 1, corresponding to the coupling topology sketched in Fig. 2. This structure implements one sign change in the coupling terms of the matrix, thanks to the change in sign of the electric field associated to the resonant mode excited in the partially filled cavity [6]. As it can be seen from the expression of $M_{3}$, this is just the necessary sign changes needed to implement this coupling matrix.

The response of the coupling matrix $M_{3}$ can now be compared with the original prototype function given by the coupling matrix $M_{1}$. Both responses are plotted together in Fig. 4. Note that the third transmission zero is not shown in this figure, since it is very far form the passband. This is due to the value of the direct coupling term, which is very small $\left(M_{S L}=0.0024\right)$. In general, the structure proposed in Fig. 1 will always exhibit small direct coupling values, so it will not be possible to approach this transmission zero to the passbands of the filter. We can observe that both responses are very similar, demonstrating that the final $M_{3}$ coupling matrix accurately corresponds to the desired dual-bandpass transfer function.

An important issue when designing filters with a new structure, is to establish the limits in the bandwidth that can be implemented from a practical point of view. Following the coupling matrix $M_{3}$ above, the lower passband is implemented by the two coupled line microstrip resonators. The bandwidth of this lower passband is controlled by the couplings from the input/output ports to these resonators $\left(M_{S 2}\right.$ and $\left.M_{3 L}\right)$. It can be observed in Fig. 1 that these couplings are implemented as standard side parallel microstrip line couplings (gaps $W_{1}=W_{3}$ in Fig. 1). Consequently, the expected maximum bandwidth is equivalent than in standard filters based on microstrip coupled line sections [11], [12].

In a similar way, the resonant mode excited in the partially filled cavity forms the upper passband of the filter. The bandwidth associated to this passband is controlled by the coupling from the input/output lines to this cavity mode $\left(M_{S 1}=-M_{1 L}\right)$. This coupling will depend on the type of substrate used, and on the length of the input/output printed lines ( $L_{\text {in }}=L_{\text {out }}$ in Fig. 1). For the dielectric substrate of our example $\left(\epsilon_{r}=2, L_{2}=3 \mathrm{~mm}\right)$, the coupling to the cavity mode 


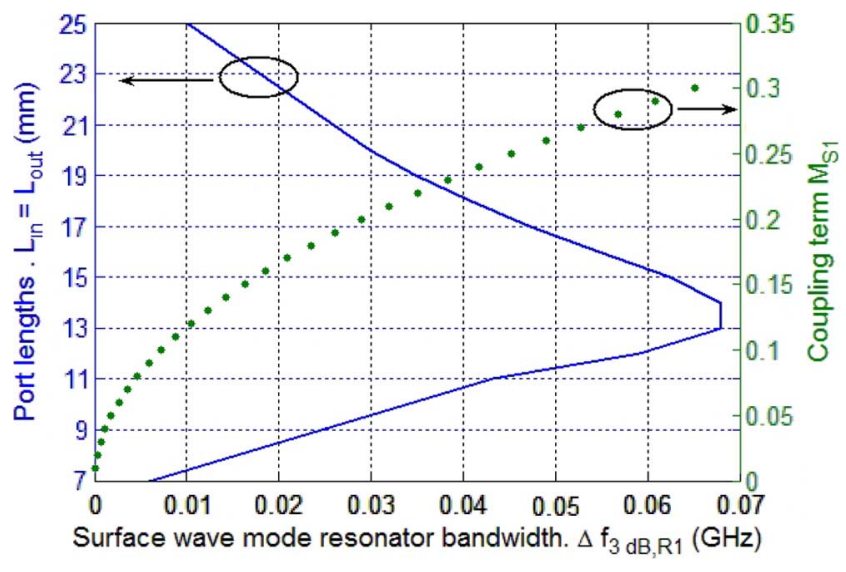

Fig. 5. Cavity mode resonator bandwidth as a function of the port lengths (left $y$-axis), and of the coupling term $M_{S 1}$ (right $y$-axis).

TABLE II

DiMENSIONS OF THE DESIGNED DUAL-BANDPASS HYBRID STRUCTURE

\begin{tabular}{c|c}
\hline Dimension & $\begin{array}{c}\text { Value } \\
\text { (in millimeters) }\end{array}$ \\
\hline \hline$a$ & 40 \\
$b$ & 34 \\
$L_{1}=L_{2}$ & 3 \\
$L_{\text {in }}=L_{\text {out }}$ & 14 \\
$L_{r 2}=L_{r 3}$ & 24.54 \\
$w_{1}=w_{3}$ & 1.8 \\
$w_{2}$ & 4.8 \\
$\varepsilon_{r}$ & 2 \\
\hline
\end{tabular}

can be extracted from the graphic shown in Fig. 5. This figure gives the $3 \mathrm{~dB}$ bandwidth of the doubly terminated resonator, as a function of the length of the input/output lines (left $y$-axis of the graphic). It is well known that this $3 \mathrm{~dB}$ bandwidth is related to the external coupling $\left(M_{S 1}\right)$ [13]. The final relation to the coupling is given in the same graphic, using the right $y$-axis. It can be seen in the graphic that the maximum coupling that can be implemented with this specific dielectric substrate is around $M_{S 1}=0.32$. Consequently, the matrix $M_{3}$ above represents a transfer function with the maximum possible bandwidth for the upper passband, that can be implemented using our structure with this specific substrate $\left(M_{S 1}=0.3091\right)$. In any case, the bandwidth of the upper passband can be easily adjusted by selecting the appropriate lengths $\left(L_{\text {in }}=L_{\text {out }}\right)$ using the data of Fig. 5. If other dielectric substrates are used in the hybrid structure, the design of the upper passband can be aided by producing a similar graphic as shown in Fig. 5, computed for the new substrate.

The dimensions of the structure after optimization for the implementation of the coupling matrix $M_{3}$ are collected in Table II. The electrical behavior of the hybrid filter can now be obtained. For this purpose, a full wave spectral domain integral equation technique for multilayered shielded structures has been used [14]. The response of the structure, together with the ideal response provided by the coupling matrix $M_{3}$ are presented in Fig. 6, showing good agreement.

Another important practical aspect when designing dual-bandpass filters is how to control the proximity of

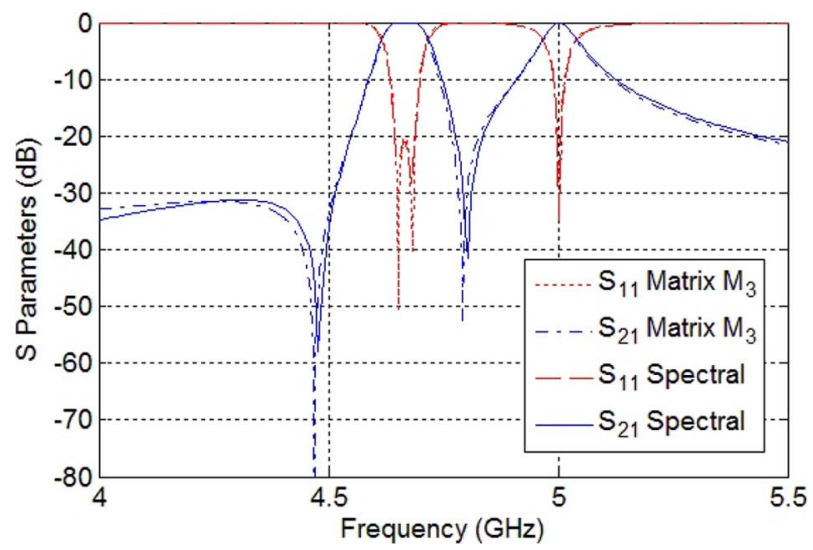

Fig. 6. Ideal model $\left(M_{3}\right)$ versus full wave simulated results of the designed hybrid structure.

the two passbands. The location of the bands can be adjusted using the diagonal elements of the matrix $M_{3}$, which give the shifts in resonant frequencies of all the resonators with respect to the center frequency of the filter. These adjustments can be implemented very easily with the new hybrid structure proposed. Since the second-order lower passband is controlled by the two coupled microstrip line resonators, their resonant frequencies can be easily controlled by acting on their lengths ( $L_{r_{2}}$ and $L_{r_{3}}$ in Fig. 1). It is worth noticing that, according to the matrix $M_{3}$, both resonators are synchronously tuned to each other $\left(M_{22}=M_{33}\right)$. Therefore, for this design the lengths of both line resonators will be equal $\left(L_{r_{2}}=L_{r_{3}}\right)$, and their value will control the position of the lower passband. Also, the level of ripple in this lower passband is controlled by the amount of coupling between these two resonators $\left(M_{23}\right)$.

In a similar way, the upper passband is controlled by the resonance associated to the sign change, which in the hybrid structure is implemented by the resonant mode excited in the partially filled cavity. The position of the upper passband, relative to the lower passband, is controlled by the diagonal element $M_{11}$. In the hybrid structure the resonant frequency of the cavity mode can be controlled with the dimensions of the shielding enclosure. A convenient way to do this is by adjusting the width of the cavity (dimension " $b$ " in Fig. 1). Consequently, by acting only on the cavity width, the upper passband can be conveniently approached to the lower passband.

To demonstrate that this is indeed the case, we present in Fig. 7 the response of the same filter as before, but for different values of the cavity width. We can observe that the lower passband is hardly affected, while the upper passband effectively approaches to the lower passband. The coupling matrices for the three filters shown in Fig. 7 are essentially the same as matrix $M_{3}$ above, expect for the element $\left(M_{11}\right)$ that changes as " $b$ " increases. Specifically, $M_{11}=-0.1414$ for $b=36 \mathrm{~mm}$, $M_{11}=-0.5714$ for $b=35 \mathrm{~mm}$, and $M_{11}=-0.9914$ for $b=34 \mathrm{~mm}$.

It is also interesting to investigate alternative transfer functions that can be implemented with the new hybrid structure proposed. One interesting alternative is to try to reverse the lower and upper passbands of the presented design. Since the resonance that changes sign takes care of the upper passband, one 


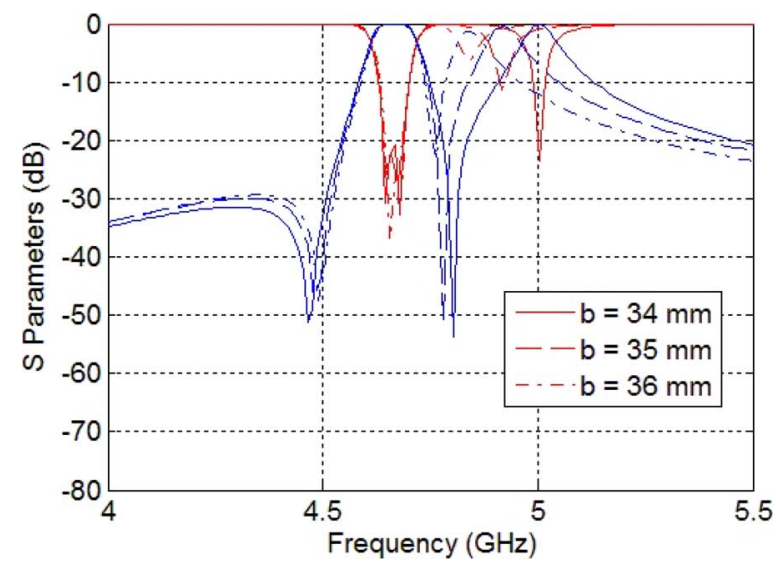

Fig. 7. Full wave simulated results of the designed hybrid structure for different values of the cavity width $(b)$, showing how the proximity of the two passbands can be controlled.

possibility is to place this resonance at a frequency lower than the other two resonances. However, with this alternative not only the bands are swapped, but also the lower transmission zero changes position, moving in between the two passbands. As a result, the two transmission zeros now combine into a pair of complex transmission zeros. This behavior can be easily shown by considering the matrix $M_{4}$

$$
M_{4}=\left(\begin{array}{rrrrr}
0 & -0.3091 & 0.4227 & 0 & 0.0024 \\
-0.3091 & 2.6200 & 0 & 0 & 0.3091 \\
0.4227 & 0 & 0.8828 & 0.2078 & 0 \\
0 & 0 & 0.2078 & 0.8828 & 0.4227 \\
0.0024 & 0.3091 & 0 & 0.4227 & 0
\end{array}\right) \text {. }
$$

This matrix is equal to our previous matrix $M_{3}$, except for the element $\left(M_{11}\right)$. This term is now the largest in the diagonal of the matrix, indicating that the resonance that changes sign is now below the two other resonances. For the hybrid structure, the implementation of this coupling matrix is easily done, by just increasing the width of the cavity to a value of $b=40 \mathrm{~mm}$. The response of the coupling matrix $M_{4}$ is plotted together with the response of the hybrid structure, in Fig. 8. We can observe that the transmission zeros are now located between the two passbands, and they are combined in the complex plane. We have also verified that the complex transmission zeros cannot be brought to the frequency axis using a symmetric network. An asymmetric network could still be used for this purpose, but it complicates the optimization tasks of the hybrid structure. In any case, this response may be useful for single-band operation and phase equalization. However, for dual-bandpass operation the high rejection properties provided by the transmission zeros in the frequency axis have been lost.

To maintain the high rejection properties between the two passbands of the filter, the location of the transmission zeros must be maintained at both sides of the second-order passband (see Fig. 4). This can be achieved in the last matrix $\left(M_{4}\right)$ by changing the sign of the inter-resonator coupling $\left(M_{23}\right)$. The response of the coupling matrix $\left(M_{4}\right)$, but with a negative value for the coupling term $\left(M_{23}\right)$ is shown in Fig. 9. In the same figure we also include the electrical performance of the original design. We can observe that the upper and lower passbands have

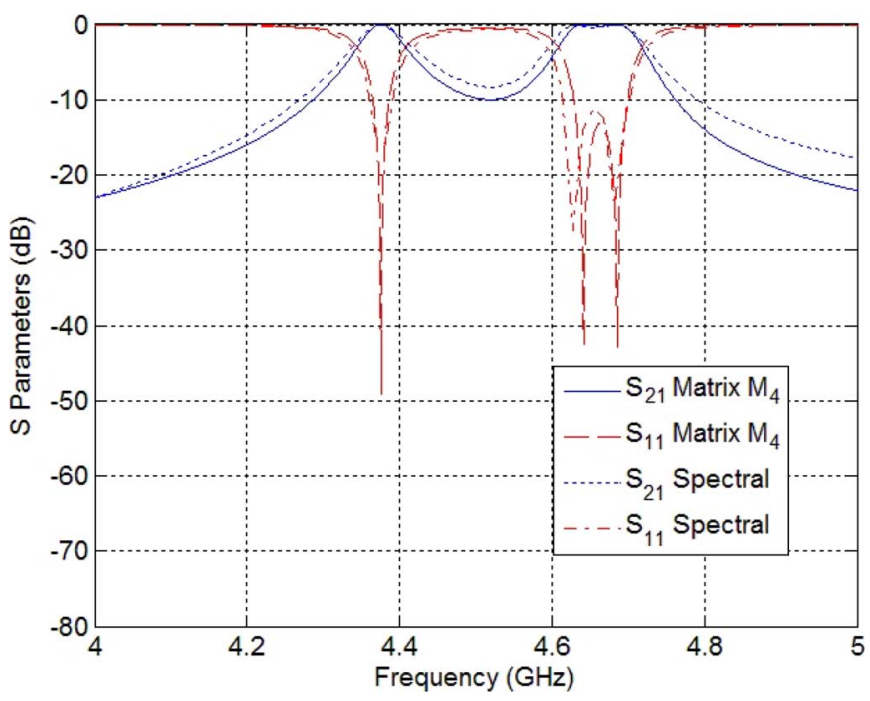

Fig. 8. Ideal model $\left(M_{4}\right)$ versus full wave simulated results of the designed hybrid structure with $b=40 \mathrm{~mm}$.

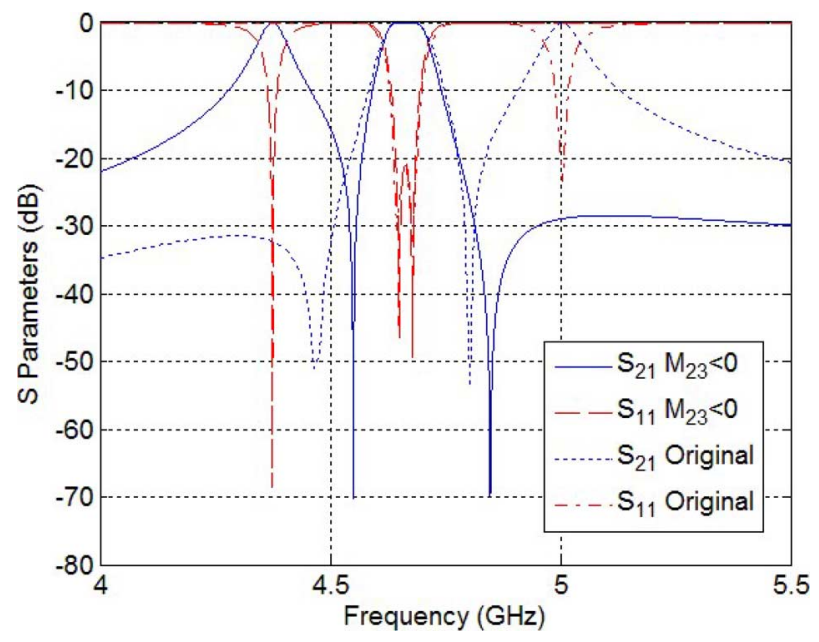

Fig. 9. Ideal model $\left(M_{4}\right.$, but with $\left.M_{23}<0\right)$ versus full wave simulated results of the original response (with $b=34 \mathrm{~mm}$ ).

been effectively swapped together, obtaining an almost perfect symmetric response with respect to the original transfer function. This time the transmission zeros appear correctly at both sides of the second-order passband, which now occupies the upper passband of the filter. However, for the practical implementation of this transfer function using the hybrid structure, a negative sign is required between the two printed line resonators. This negative coupling between the printed line resonators cannot be implemented with the proposed configuration, where simple half-wavelength printed resonators are proposed. A more complicated layout employing open-loop resonators could be used to implement this negative coupling (see, e.g., [15]).

Finally, higher order dual-band filters can also be implemented by employing the hybrid technology under study. A possible extension is obtained just by adding an additional microstrip resonator between the input/output ports in Fig. 1. This new structure will correspond to the coupling topology shown in Fig. 10, where in the upper branch the three printed 


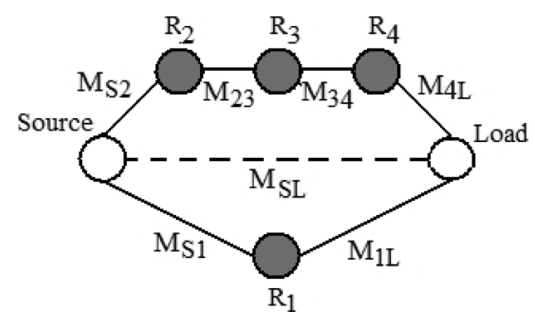

Fig. 10. Coupling scheme of a fourth-order fully canonical filter. Again, $M_{S L}$ represents the direct coupling from source to load.

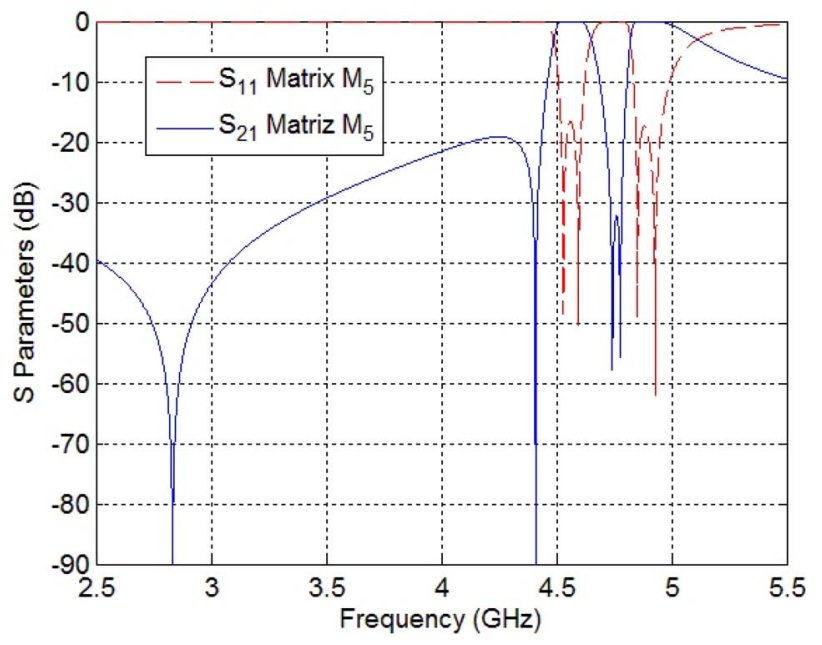

Fig. 11. Response of the coupling matrix $M_{5}$.

line resonators are coupled inline. However, a limitation of the hybrid structure is that the cavity mode is always directly coupled to both the input and the output ports. Consequently, it is not possible to couple more resonators, in inline configuration, in the lower branch of Fig. 10.

In spite of this limitation, it is possible to synthesize a dualbandpass response with the topology shown in Fig. 10. In this case each passband has two reflection zeros, and two transmission zeros are used to separate the two passbands of the filter. Being a fully canonical filter, the total number of transmission zeros that can be implemented at finite frequencies is four. A coupling matrix that satisfies all these requirements is

$$
M_{5}=\left(\begin{array}{rrrrrr}
0 & -0.575 & 0.533 & 0 & 0 & 0.027 \\
-0.575 & -1.095 & 0 & 0 & 0 & 0.575 \\
0.533 & 0 & 0.483 & 0.511 & 0 & 0 \\
0 & 0 & 0.511 & 0.084 & 0.511 & 0 \\
0 & 0 & 0 & 0.511 & 0.484 & 0.533 \\
0.027 & 0.575 & 0 & 0 & 0.533 & 0
\end{array}\right) .
$$

The response of this coupling matrix is presented in Fig. 11. We can observe from the response that the prototype presents a total bandwidth of $450 \mathrm{MHz}$, centered at $4.7 \mathrm{GHz}$. The filtering response is composed of two second-order passbands with a total of four transmission zeros. Two transmission zeros are located to the left of the lower passband, whereas the two remaining transmission zeros are located between the two passbands.

Note that it is possible to implement this coupling matrix with the hybrid structure proposed in this paper, since all the couplings are positive, apart from the $M_{S 1}$ element, which is
TABLE III

Dimensions OF THE MANUFACTURED DUAL-BANDPASS FILTER IN HYBRID TECHNOLOGY

\begin{tabular}{c|c}
\hline Dimension & $\begin{array}{c}\text { Value } \\
\text { (in millimeters) }\end{array}$ \\
\hline \hline$a$ & 40 \\
$b$ & 34 \\
$L_{1}$ & 3 \\
$L_{2}$ & 3.14 \\
$L_{\text {in }}=L_{\text {out }}$ & 14 \\
$L_{r} 2=L_{r} 3$ & 24.54 \\
$w_{1}=w_{3}$ & 1.8 \\
$w_{2}$ & 5 \\
$\varepsilon_{r}$ & 2.20 \\
\hline
\end{tabular}

implemented by the cavity resonance. All the other three resonances can be implemented by coupling, in inline configuration, three printed line resonators of the same type, as shown in Fig. 1. From the matrix $M_{5}$, we also see that the three resonators coupled in the upper branch are not anymore synchronously tuned, since the diagonal term $M_{33}$ is different from the diagonal terms associated to the other resonances $\left(M_{22}\right.$ and $\left.M_{44}\right)$.

\section{EXPERIMENTAL IMPLEMENTATION}

In this section, a third-order example has been designed, manufactured and tested, in order to provide experimental verification of the novel structure. The substrate selected for manufacturing is an RT Duroid with relative permittivity $\varepsilon_{r}=2.2$ and thickness of $1.57 \mathrm{~mm}$. The prototype implements a dual bandpass filter with $-17 \mathrm{~dB}$ of return loss, and three transmission zeros at $1.4,4.3$, and $4.6 \mathrm{GHz}$. The center frequency is $4.7 \mathrm{GHz}$ and its bandwidth is $430 \mathrm{MHz}$. The $(N+2)$ coupling matrix corresponding to the topology shown in Fig. 2 results to be

$$
M_{6}=\left(\begin{array}{rrrrr}
0 & -0.2895 & 0.3721 & 0 & 0.0024 \\
-0.2895 & -0.9804 & 0 & 0 & 0.2895 \\
0.3721 & 0 & 0.8512 & 0.1800 & 0 \\
0 & 0 & 0.1800 & 0.8512 & 0.3721 \\
0.0024 & 0.2895 & 0 & 0.3721 & 0
\end{array}\right) .
$$

The dimensions of the designed and manufactured dual-bandpass filter, obtained after optimization, are shown in Table III. For the manufactured prototype, two RT Duroid substrates are piled up together to obtain the total required dielectric height of $L_{2}=3.14 \mathrm{~mm}$. This extended thickness is needed to obtain the required high coupling value $\left(M_{S 1}=-0.2895\right)$ to the cavity mode.

In Fig. 12, we present the results obtained from the analysis of the structure, and we compare them with the predicted response of the $M_{6}$ matrix. We can observe good agreement between both responses. In addition, we present in Fig. 13 the measured results of the manufactured prototype, and we compare them with simulated results using the full wave spectral domain integral equation technique reported in [14]. In the simulations, losses are included in the dielectric substrate $(\tan (\delta)=0.003)$, and in the printed metalizations $\left(\sigma=1 \cdot 10^{7} \Omega^{-1} / \mathrm{m}\right)$. However, losses in the shielding cavity were not included due to limitations of the software. It can be observed that the simulated and measured results are in good agreement. The minimum insertion loss of the filter inside the lower passband is $-1.3 \mathrm{~dB}$, whereas inside the upper passband the minimum insertion loss increases 


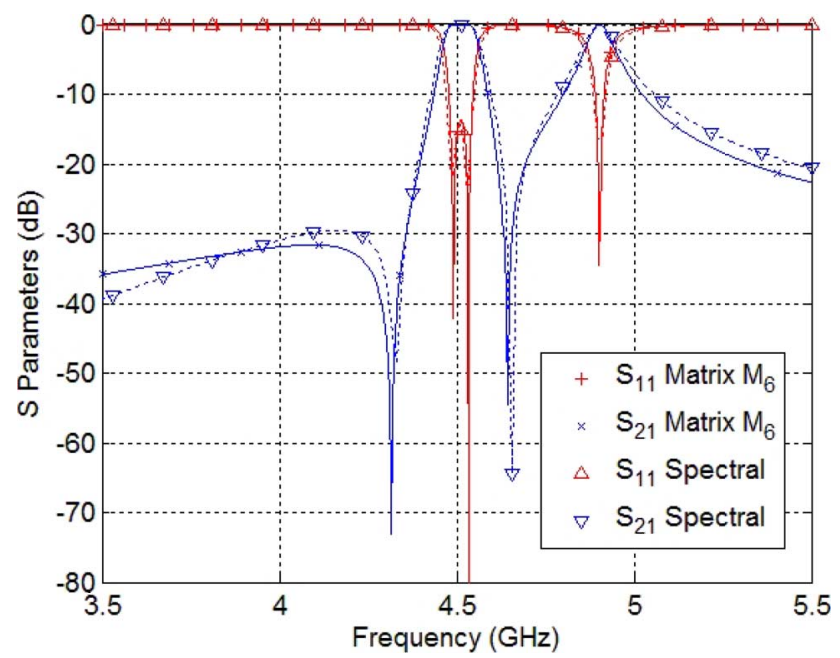

Fig. 12. Comparison of ideal model and full wave lossless simulation results.

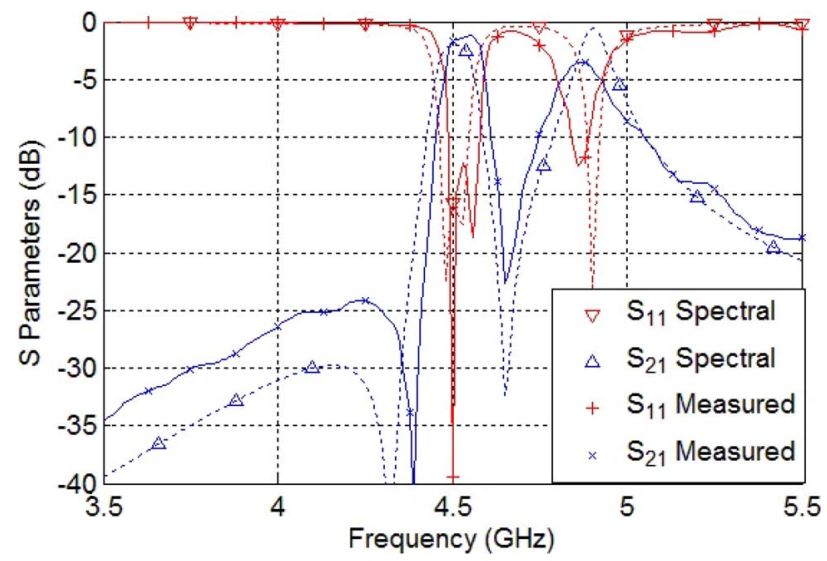

Fig. 13. Comparison between simulated results with losses $(\tan (\delta)=0.003$, $\left.\sigma=1 \cdot 10^{7} \Omega^{-1} / \mathrm{m}\right)$, and measured results.

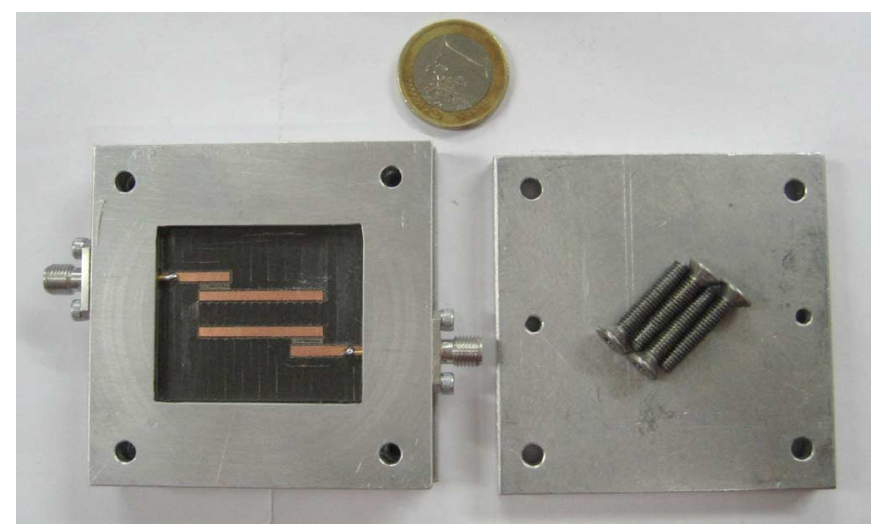

Fig. 14. Fabricated prototype showing all the pieces of the filter.

to $-3.45 \mathrm{~dB}$. The high insertion losses in the upper passband are due to the use of a low quality brass material for the manufacturing of the cavity box. As already said, the upper passband is formed by a resonant mode excited in this cavity. Therefore, the conductivity of the cavity strongly influences the insertion losses of the upper passband. Consequently, the high insertion losses in the upper passband can be reduced using silver plating techniques on the walls of the shielding cavity.
Due to the relative large bandwidth of this design, the resulting topology does not exhibit high sensitivity to mechanical tolerances. The mechanical precision achieved during manufacturing was of $\pm 0.05 \mathrm{~mm}$. With these mechanical tolerances, the agreement between measured results and simulations is reasonable. A photograph of the manufactured prototype is shown in Fig. 14.

\section{CONCLusions}

A dual-bandpass filtering structure with a specific coupling topology has been proposed. The filter configuration, which provides dual-bandpass operation, has been implemented by means of a novel hybrid waveguide-microstrip technology. Important aspects about the filter design, useful for practical applications, have been highlighted throughout the paper. Two designs have been illustrated in order to show the validity of the proposal. Finally, one of the designs has been manufactured and tested, showing good agreement with respect to the predicted results.

\section{REFERENCES}

[1] G. Macchiarella and S. Tamiazzo, "Design techniques for dual-passband filters," IEEE Trans. Microw. Theory Tech., vol. 53, no. 11, pp. 3256-3271, Nov. 2005.

[2] R. J. Cameron, M. Yu, and Y. Wang, "Direct-coupled microwave filters with single and dual stopbands," IEEE Trans. Microw. Theory Tech., vol. 53, no. 11, pp. 3288-3297, Nov. 2005.

[3] M. Mokhtaari, J. Bornemann, K. Rambabu, and S. Amari, "Couplingmatrix design of dual and triple passband filters," IEEE Trans. Microw. Theory Tech., vol. 54, no. 11, pp. 3940-3946, Nov. 2006.

[4] R. J. Cameron, J. C. Faubere, and F. Seyfert, "Coupling matrix synthesis for a new class of microwave configuration," in IEEE MTT-S Int. Microw. Symp. Dig., Long Beach, CA, Jun. 12-17, 2005, pp. 119-122.

[5] V. Lunot, F. Seyfert, S. Bila, and A. Nasser, "Certified computation of optimal multiband filtering functions," IEEE Trans. Microw. Theory Tech., vol. 56, no. 1, pp. 105-112, Jan. 2008.

[6] M. Martínez-Mendoza, J. S. Gómez-Díaz, D. C. Rebenaque, J. L. Gómez-Tornero, and A. Alvarez-Melcon, "Design of a bandpass transversal filter employing a novel hybrid printed structure," in IEEE MTT-S Int. Microw. Symp. Dig., Honolulu, HI, Jun. 6-11, 2007, pp. 1281-1284.

[7] R. J. Cameron, "Advanced coupling matrix synthesis techniques for microwave filters," IEEE Trans. Microw. Theory Tech., vol. 51, no. 1, pp. 1-10, Jan. 2003.

[8] S. Amari, U. Rosenberg, and J. Borneman, "Adaptive synthesis and design of resonator filters with source/load-multiresonator coupling," IEEE Trans. Microw. Theory Tech., vol. 50, no. 8, pp. 1969-1975, Aug. 2002.

[9] S. Amari, U. Rosenberg, and J. Borneman, "Synthesis of cross-coupled resonator filters using an analytical gradient-based optimization technique," IEEE Trans. Microw. Theory Tech., vol. 48, no. 9, pp. 1559-1564, Sep. 2000.

[10] R. J. Cameron, "General coupling matrix synthesis method for chebyshev filtering functions," IEEE Trans. Microw. Theory Tech., vol. 47, no. 4, pp. 433-442, Apr. 1999.

[11] S. B. Cohn, "Parallel-coupled transmission-line-resonator filters," IEEE Trans. Microw. Theory Tech., vol. 6, no. 2, pp. 223-231, Apr. 1958.

[12] G. Matthaei and E. Yones, Microwave Filters, Impedance Matching Networks, and Coupling Structures. Boston, MA: Artech House, 1980.

[13] D. Swanson and G. Macchiarella, "Microwave filter design by synthesis and optimization," IEEE Micro, pp. 55-69, Apr. 2007.

[14] A. A. Melcon, J. R. Mosig, and M. Guglielmi, "Efficient CAD of boxed microwave circuits based on arbitrary rectangular elements," IEEE Trans. Microw. Theory Tech., vol. 47, no. 7, pp. 1045-1058, Jul. 1999.

[15] D. C. Rebenaque, F. Q. Pereira, J. P. Garcia, A. A. Melcon, and M. Guglielmi, "Two compact configurations for implementing transmission zeros in microstrip filters," IEEE Microw. Wireless Compon. Lett., vol. 14 , no. 10 , pp. $475-477$, Oct. 2004. 


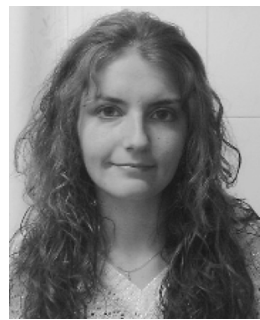

Mónica Martínez-Mendoza (S'07) was born in Cartagena, Murcia, Spain, in 1983. She received the Telecommunications Engineer degree from the Technical University of Cartagena (UPCT), Cartagena, Spain, in 2006, and is currently working toward the Ph.D. degree at UPCT.

In 2007, she joined the Telecommunications and Electromagnetic Group, UPCT, as a Research Assistant, where she is involved in the development of novel transversal filtering structures for satellite systems. In September 2008, she joined the European Space Agency (ESA), European Space Research and Technology Centre (ESTEC), Noordwijk, The Netherlands, where she is involved in the design of microwave filters and multiplexers for space applications. Her current scientific interests include the analysis and design of microwave circuits.

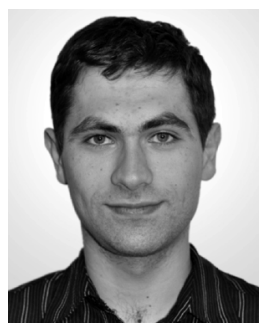

Juan Sebastián Gómez-Díaz (S'07) was born in Albacete, Spain, in 1983. He received the Telecommunications Engineer degree (with honors) from the Technical University of Cartagena (UPCT), Cartagena, Spain, in 2006, and is currently working toward the Ph.D. degree at UPCT.

In 2007 he has joined the Telecommunication and Electromagnetic Group (GEAT), UPCT, as a Research Assistant. From November 2007 to October 2008, he was a visiting Ph.D. student with the Poly-Grames, École Polytechnique de Montréal, where he was involved in the impulse-regime analysis of linear and nonlinear metamaterial-based devices and antennas. His current scientific interests include integral equations and numerical methods and their application to the analysis and design of microwave circuits and antennas.

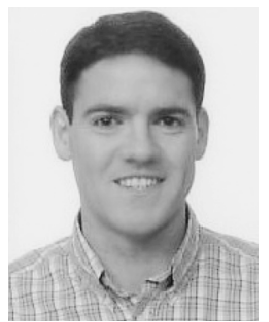

David Cañete-Rebenaque (S'04) was born in Valencia, Spain, in 1976. He received the Telecommunications Engineer degree from the Technical University of Valencia, Valencia, Spain, in 2000, and is currently working toward the Ph.D. degree at the University of Cartagena, Cartagena, Spain.

During 2001, he was an RF Engineer with a mobile communication company. In 2002, he joined the Communications and Information Technologies Department, Technical University of Cartagena (UPCT). His research interests include analysis and design of microwave circuits and active antennas.

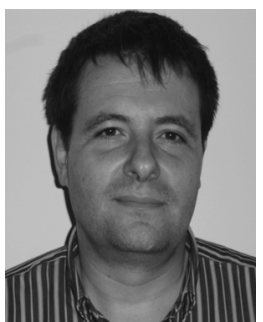

Alejandro Alvarez-Melcón (M'99-SM'07) was born in Madrid, Spain, in 1965. He received the Telecommunications Engineer degree from the Technical University of Madrid (UPM), Madrid, Spain, in 1991, and the Ph.D. degree in electrical engineering from the Swiss Federal Institute of Technology, Lausanne, Switzerland, in 1998.

In 1988, he joined the Signal, Systems and Radiocommunications Department, UPM, as a Research Student, where he was involved in the design, testing, and measurement of broadband spiral antennas for electromagnetic measurements support (EMS) equipment. From 1991 to 1993, he was with the Radio Frequency Systems Division, European Space Agency (ESA)/European Space Research and Technology Centre (ESTEC), Noordwijk, The Netherlands, where he was involved in the development of analytical and numerical tools for the study of waveguide discontinuities, planar transmission lines, and microwave filters. From 1993 to 1995, he was with the Space Division, Industry Alcatel Espacio, Madrid, Spain, and also with the ESA, where he collaborated on several ESA/ESTEC contracts. From 1995 to 1999, he was with the Swiss Federal Institute of Technology, École Polytechnique Fédérale de Lausanne (EPFL), Lausanne, Switzerland, where he was involved in the field of microstrip antennas and printed circuits for space applications. In 2000, he joined the Technical University of Cartagena (UPCT), Cartagena, Spain, where he currently develops his teaching and research activities.

Dr. Alvarez-Melcón was the recipient of the Journée Internationales de Nice Sur les Antennes (JINA) Best Paper Award for the best contribution to the JINA98 International Symposium on Antennas, and the Colegio Oficial de Ingenieros de Telecomunicación (COIT/AEIT) Award for the best doctoral thesis in basic information and communication technologies. 